\title{
Особенности роста
}

\section{макроаденом гипофиза}

с различной гормональной

\section{актИВНОстью}

Иловайская И.А. • Древаль А.В. • Кривошеева Ю.Г. • Астафьева Л.И. • Сташук Г.А. ${ }^{1}$

Иловайская Ирэна Адольфовна канд. мед. наук, ст. науч. сотр., отделение терапевтической эндокринологии'

129110, г. Москва, ул. Щепкина, 61/2-9, Российская Федерация.

Тел.: +7 (495) 6317492.

E-mail: irena.ilov@yandex.ru

Древаль Александр Васильевич - д-р мед. наук, профессор, руководитель отделения терапевтической эндокринологии, заведующий кафедрой эндокринологии факультета усовершенствования врачей ${ }^{1}$

Кривошеева Юлия Григорьевна врач-эндокринолог, аспирант кафедры эндокринологии факультета усовершенствования врачей

Астафьева Людмила Игоревна - д-р мед. наук, вед. науч. сотр., отделение опухолей гипофиза

Сташук Галина Александровна д-р мед. наук, гл. науч. сотр. рентгенологического отделения, профессор кафедры лучевой диагностики факультета усовершенствования врачей
Актуальность. Аденома гипофиза - не столь редкое заболевание, как представлялось ранее. Частота макроаденом в общей популяции составляет до 0,16-0,2\%. Магнитно-резонансная томография (МРТ) является методом выбора диагностики опухолей гипофиза. До сих пор не обсуждались особенности визуализации опухолей гипофиза с различной гормональной активностью. Цель - провести сравнительный анализ размера, объема и направления роста у макроаденом гипофиза с различной гормональной активностью. Материал и методы. Анализировались данные МРТ 305 пациентов с аденомами гипофиза размером более 10 мм: с гормонально неактивными аденомами гипофиза $(n=109)$, пролактиномами $(n=58)$ и соматотропиномами $(n=138)$. Результаты. В зависимости от гормональной активности аденомы гипофиза различались по объему ( $<$ <0,001): гормонально неактивные аденомы гипофиза были 6620 [2637; 14492] мм³, пролактиномы - 5365 [1495; 10316] мм³ соматотропиномы - 3052 [1696; 5727] мм³. У большинства больных всех групп наблюдался экстраселлярный рост в нескольких направлениях. Рост в одном направлении отмечался среди 29\% гормонально неактивных аденом гипофиза, $41 \%$ пролактином и $37 \%$ соматотропином ( $>0,05)$. Преобладающим среди гормонально неактивных аденом гипофиза и пролактином был супраселлярный рост (в 83,5 и 79,3\% случаев соответственно), тогда как среди соматотропином превалировало инфраселлярное распространение (66,1\%). Заключение. Данные особенности макроаденом гипофиза с различной гормональной активностью могут быть использованы для дифференциальной диагностики, что будет способствовать оптимизации обследования больных на этапах диагностического поиска.

Ключевые слова: макроаденомы гипофиза, гормонально неактивные аденомы гипофиза, пролактиномы, соматотропиномы, направление роста макроаденом гипофиза

\section{doi: 10.18786/2072-0505-2016-44-4-451-456}

'ГБУЗ МО «Московский областной научно-исследовательский клинический институт им. М.Ф. Владимирского»; 129110, г. Москва, ул. Щепкина, 61/2, Российская Федерация

2 ФГАУ «Научно-исследовательский институт нейрохирургии имени академика Н.Н. Бурденко» Минздрава России; 125047, г. Москва, ул. 4-я Тверская-Ямская, 16, Российская Федерация 
A деномы гипофиза - доброкачественные опухоли, развивающиеся из железистой ткани передней доли гипофиза. Их распространенность составляет 10-15\% от всех диагностированных прижизненно опухолей головного мозга и $17-25 \%$ от всех внутричерепных новообразований согласно данным патологоанатомических исследований [1].

По размеру аденомы гипофиза условно подразделяют на микроаденомы (размерами 10 мм и менее) и макроаденомы (более 10 мм). Метаанализ 10 крупных скрининговых прижизненных (радиологических) и постмортальных исследований показал, что частота макроаденом в общей популяции достигает 0,16-0,2\% [2]. В последних эпидемиологических исследованиях распространенность аденом гипофиза оказалась примерно в 3-5 раз выше, чем представлялось ранее [3-5]. Таким образом, аденомы гипофиза не столь редкое заболевание, как принято считать.

Макроаденомы гипофиза по сравнению с микроаденомами ставят перед клиницистами более сложные задачи: на этапе диагностики необходимо оценивать не только гормональную активность аденомы, но и степень ее распространения за пределы турецкого седла, а в процессе лечения - контролировать не только гормональную продукцию, но и объем образования.

Магнитно-резонансная томография (MPT) считается методом выбора диагностики опухолей гипофиза [5-7]. Возможность проведения исследования в трех взаимно перпендикулярных проекциях (сагиттальной, вертикальной и фронтальной) позволяет получить информацию об анатомо-топографических особенностях и изменениях селлярной области, проводить четкую дифференциацию между пролиферативными процессами в гипофизе и другими изменениями. Среди всех изменений, выявляемых в области турецкого седла, наиболее часто встречаются гормонально неактивные опухоли гипофиза, пролактиномы и соматотропиномы [6, 7]. До сих пор накоплено недостаточно данных об особенностях аденом гипофиза с различной гормональной активностью.

Цель - провести сравнительный анализ размера, объема и направления роста у макроаденом гипофиза с различной гормональной активностью.

\section{Материал и методы}

В исследование вошли пациенты, находившиеся под наблюдением в ГБУЗ МО «Московский областной научно-исследовательский клинический институт им. М.Ф. Владимирского» с 2004 по 2015 г. Были проанализированы данные МРТ 305 пациентов с макроаденомами гипофиза до проведения какого-либо лечения. Из них 109 человек были с гормонально неактивными аденомами гипофиза, 58 - с пролактиномами и 138 - с соматотропиномами. Диагностика гормональной активности опухолей гипофиза основывалась на совокупности клинической картины и гормональных изменений: для гормонально неактивных аденом гипофиза это были отсутствие признаков гиперсекреции гормонов аденогипофиза и/или гистологическое заключение после нейрохирургического удаления опухоли; для пролактином - гиперпролактинемия выше 3000 мМЕ/мл и/или значимое уменьшение размеров опухоли в ответ на последующее лечение агонистами дофамина; для соматотропином - увеличение уровня инсулиноподобного фактора роста 1 выше половозрастных значений и пиковое значение уровня соматотропного гормона в ходе теста с нагрузкой глюкозой более 1 нг/мл. Характеристика пациентов, включенных в исследование, представлена в табл. 1 .

Возраст пациентов в группах статистически значимо различался $(\mathrm{p}<0,001)$. Пациенты с гормонально неактивными аденомами гипофиза были старше больных с пролактиномами. Во всех группах превалировали женщины.

MPТ головного мозга с прицельным исследованием гипофиза выполнялась на высокопольном аппарате InteraAchieva (Philips) со сверхсильной напряженностью магнитного поля 3 Тл, с внутривенным введением внеклеточных гадолинийсодержащих контрастных препаратов. Оценивали максимальный линейный размер в вертикальной, сагиттальной (переднезадней) и фронтальной (поперечной) плоскостях, единица измерения - миллиметр. Для вычисления

Таблица 1. Характеристика пациентов с макроаденомами гипофиза различной гормональной активности

\begin{tabular}{|c|c|c|c|}
\hline \multirow[t]{2}{*}{ Параметр } & \multicolumn{3}{|l|}{ Группа } \\
\hline & $\begin{array}{l}\text { гормонально } \\
\text { неактивные аденомы } \\
\text { гипофиза }\end{array}$ & пролактиномы & соматотропиномы \\
\hline $\begin{array}{l}\text { Количество пациентов, } \\
\text { абс. }\end{array}$ & 109 & 58 & 138 \\
\hline Возраст пациентов, годы* & $25-80 ; 59[52 ; 66]$ & $20-79 ; 47[34 ; 47]$ & $23-80 ; 55[43 ; 64]$ \\
\hline $\begin{array}{l}\text { Количество мужчин/ } \\
\text { женщин, \% }\end{array}$ & $30 / 70$ & $35 / 66$ & $21 / 79$ \\
\hline
\end{tabular}

“Данные представлены в виде минимума и максимума (Min-max); медианы (Me) и 25-го и 75-го перцентиля [25; 75] 
Таблица 2. Размеры макроаденом гипофиза с различной гормональной активностью (по данным магнитно-резонансной томографии)

\begin{tabular}{|c|c|c|c|c|}
\hline \multirow[t]{2}{*}{ Размер опухоли, мм } & \multicolumn{3}{|l|}{ Группа } & \multirow[t]{2}{*}{ Значение $p$} \\
\hline & $\begin{array}{l}\text { гормонально неактивные } \\
\text { аденомы гипофиза } \\
\text { (1) }\end{array}$ & $\begin{array}{l}\text { пролактиномы } \\
\text { (2) }\end{array}$ & $\begin{array}{l}\text { соматотропиномы } \\
\text { (3) }\end{array}$ & \\
\hline Вертикальный & $\begin{array}{l}8-51 ; 24[17,5 ; 34,5] \\
p^{*}<0,001\end{array}$ & $9-65 ; 21[15 ; 31]$ & $5-61 ; 18[14 ; 25]$ & $\begin{array}{l}\mathrm{p}_{1-2}=0,213 \\
\mathrm{p}_{1-3}<0,001 \\
\mathrm{p}_{2-3}=0,006\end{array}$ \\
\hline Сагиттальный & $\begin{array}{l}9-56 ; 23[17 ; 28] \\
\mathrm{p}^{*}<0,005\end{array}$ & $12-62 ; 20[16 ; 30]$ & $8-67 ; 18[14 ; 24]$ & $\begin{array}{l}\mathrm{p}_{1-2}=0,954 \\
\mathrm{p}_{1-3}=0,001 \\
\mathrm{p}_{2-3}=0,2\end{array}$ \\
\hline Фронтальный & $\begin{array}{l}8-62 ; 23[18 ; 30] \\
\mathrm{p}^{*}<0,001\end{array}$ & $10-62 ; 20[14 ; 30]$ & $5-60 ; 17[15 ; 23]$ & $\begin{array}{l}\mathrm{p}_{1-2}=0,391 \\
\mathrm{p}_{1-3}<0,001 \\
\mathrm{p}_{2-3}=0,031\end{array}$ \\
\hline
\end{tabular}

Данные представлены в виде минимума и максимума (Min-max); медианы (Me) и 25-го и 75-го перцентиля [25; 75]

p - для попарного сравнения групп

p* - для множественного сравнения групп

объема опухоли гипофиза использовалась следующая формула: $\mathrm{ABC} \times \pi \times 4 / 3$, где $\mathrm{ABC}$ - размеры полуосей (сагиттальной, вертикальной и фронтальной) опухоли. Наряду с этим характеризовали частоту и характер распространения опухоли за пределы турецкого седла.

Статистическая обработка полученных результатов проводилась с использованием программы Statistica 10.0 для Windows 7 с применением методов вариационной статистики для непараметрических данных. Результаты представлены в виде медианы (Ме) и интерквартильного размаха [Q25; Q75]. Для проведения корреляционного анализа использовался метод Спирмена. Для определения статистической значимости различий в независимых группах применяли метод Крускала - Уоллиса для множественного сравнения и U-тест Манна - Уитни с поправкой Бонферрони для попарного сравнения групп.

\section{Результаты и обсуждение}

В нашей когорте пациентов опухоли гипофиза с различной гормональной активностью различались по всем параметрам. Наибольшими размерами обладали гормонально неактивные макроаденомы гипофиза (табл. 2). Тем не менее при попарном сравнении групп статистически значимых различий между гормонально неактивными макроаденомами гипофиза и макропролактиномами выявлено не было. Размеры гормонально неактивных макроаденом гипофиза по всем параметрам были статистически значимо больше размеров макросоматотропином. Вертикальный и фронтальный размеры макропролактином были больше по сравнению с макросоматотропиномами, при этом сагиттальные размеры у этих видов опухолей гипофиза не различались.

Объемы макроаденом гипофиза с различной гормональной активностью также статистически значимо различались $(\mathrm{p}<0,001)$ (рис. 1). Интересно отметить, что индивидуальные максимальные показатели объема опухолей гипофиза наблюдались среди пролактином и соматотропином: если среди гормонально неактивных макроаденом гипофиза максимальный объем новообразования составил 68753 мм³ $^{3}$ то среди пролактином - $130760 \mathrm{~mm}^{3}$, а соматотропином 124501 мм $^{3}$. Однако в целом по группам наибольший объем наблюдался у гормонально неактивных макроаденом гипофиза - 6620 [2637; 14492] мм $^{3}$, несколько меньше он был у пролактином 5365 [1495; 10316] мм³ , и еще более низкие показатели отмечались у соматотропином - 3052 [1696; 5727] мм³. При этом не было выявлено статистически значимых различий между гормонально неактивными макроаденомами гипофиза и пролактиномами $(\mathrm{p}=0,204)$, а также пролактиномами и соматотропиномами $(\mathrm{p}=0,14)$, но объем гормонально неактивных макроаденом гипофиза был статистически значимо больше объема соматотропином $(\mathrm{p}<0,001)$.

Группы макроаденом гипофиза различались и по направлениям роста (рис. 2). Супраселлярный рост угормонально неактивных макроаденом гипофиза встречался статистически значимо чаще, чем у пролактином ( $<0,001)$, в свою очередь у пролактином - статистически значимо чаще, чем у соматотропином $(\mathrm{p}<0,001)$. Преобладающим направлением роста 


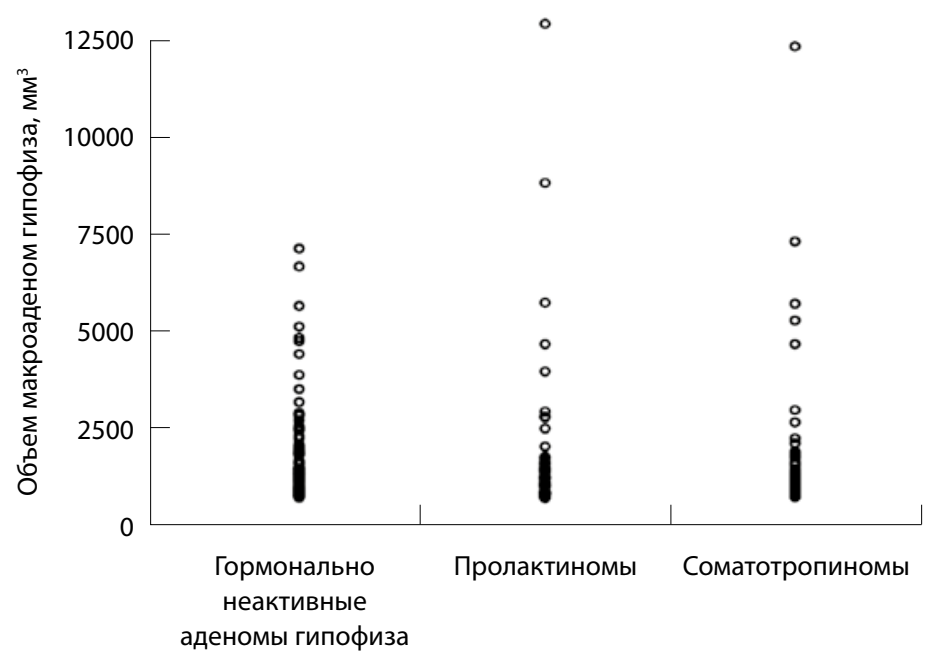

Рис. 1. Объемы макроаденом гипофиза с различной гормональной активностью

у гормонально неактивных макроаденом гипофиза и пролактином был именно супраселлярный рост, тогда как у соматотропином преобладало инфраселлярное распространение $(\mathrm{p}<0,001$ по сравнению с гормонально неактивными макроаденомами гипофиза и пролактиномами). Частота инфраселлярного роста у гормонально неактивных макроаденом гипофиза и пролактином статистически значимо не различалась $(\mathrm{p}=0,449)$. Отмечалась несколько более высокая частота латероселлярного роста у соматотропином по сравнению с гормонально неактивными макроаденомами гипофиза и пролактиномами, однако различия не достигли уровня статистической значимости $(\mathrm{p}=0,81)$.

Компрессия хиазмы по данным МРТ отмечалась в 55\% случаев среди гормонально неактивных макроаденом гипофиза, в 35\% - среди пролактином и $19 \%$ - среди соматотропином ( $<<0,001)$, что коррелировало с вертикальным размером и объемом опухолей.

Эндоселлярные макроаденомы гипофиза встречались всего лишь в 4\% наблюдений в группе пациентов с гормонально неактивными макроаденомами гипофиза, в $4 \%$ - среди пролактином и в 7,8\% - в группе пациентов с соматотропиномами. Во всех группах для большинства макроаденом было характерно экстраселлярное распространение, среди гормонально неактивных макроаденом гипофиза и пролактином превалировал супраселлярный рост, а среди соматотропином - инфраселлярный (см. рис. 2). Экстраселлярный рост только в одном направлении отмечался среди 29\% гормонально неактивных макроаденом гипофиза, 41\% пролактином

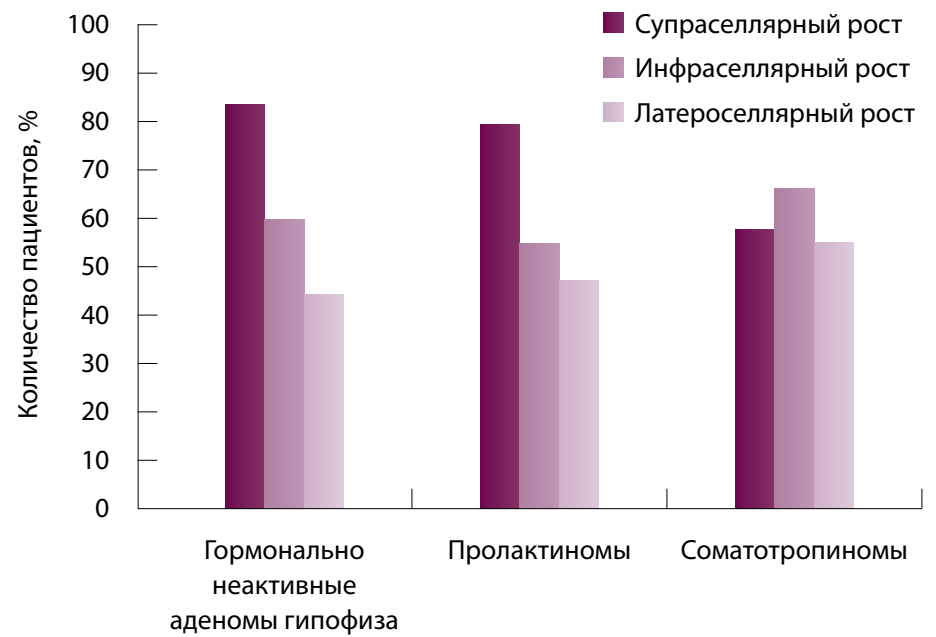

Рис. 2. Направления роста макроаденом гипофиза с различной гормональной активностью

и $37 \%$ соматотропином $(\mathrm{p}=0,092)$. При этом та же тенденция сохранялась и в отношении частоты направлений экстраселлярного роста - у гормонально неактивных макроаденом гипофиза и пролактином наиболее часто отмечался супраселлярный рост, а среди соматотропином - инфраселлярный (рис. 3-5).

Работ по оценке объема опухолей гипофиза немного. В серии МРТ головного мозга, проведенных в условиях специализированного отделения ФГБУ «Эндокринологический научный центр» Минздрава России, было показано, что средний объем гормонально неактивных опухолей гипофиза составляет $2863 \mathrm{~mm}^{3}$, соматотропином $2470 \mathrm{mм}^{3}$, пролактином - $1362 \mathrm{mм}^{3}$ [8]. Однако в это исследование были включены как макро-, так и микроаденомы гипофиза. Известно, что соотношение микро- и макроаденом гипофиза различается для каждого вида опухоли гипофиза:

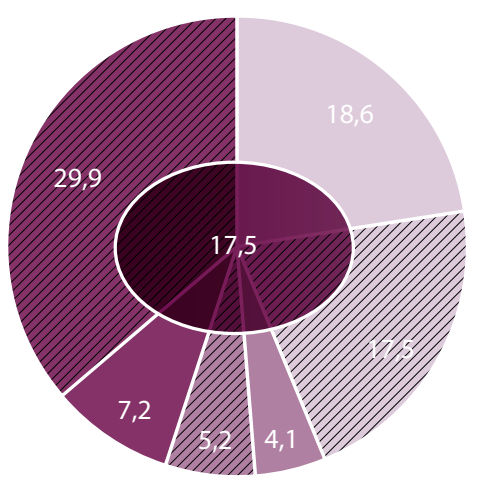

Супраселлярный рост

V/, Супраселлярный рост + латероселлярный рост

- Латероселлярный рост

घ/. Латероселлярный рост + инфраселлярный рост

- Инфраселлярный рост

Инфраселлярный рост + супраселлярный рост

- Супраселлярный рост + латероселлярный рост + инфраселлярный рост

Рис. 3. Частота разных направлений экстраселлярного роста среди гормонально неактивных аденом гипофиза, \% 


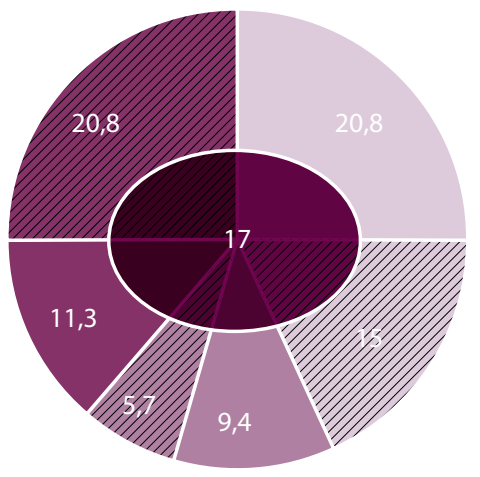

Супраселлярный рост

Y/, Супраселлярный рост + латероселлярный рост

Латероселлярный рост

$\mathbb{Z}$ Латероселлярный рост + инфраселлярный рост

Инфраселлярный рост

च Инфраселлярный рост + супраселлярный рост

Супраселлярный рост + латероселлярный рост + инфраселлярный рост

Рис. 4. Частота разных направлений экстраселлярного роста среди пролактином, \%

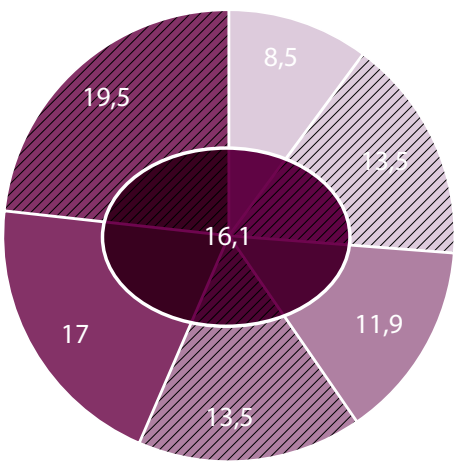

Супраселлярный рост

Y/. Супраселлярный рост + латероселлярный рост

Латероселлярный рост

प/. Латероселлярный рост + инфраселлярный рост

Инфраселлярный рост

Инфраселлярный рост + супраселлярный рост

Супраселлярный рост + латероселлярный рост + инфраселлярный рост

Рис. 5. Частота разных направлений экстраселлярного роста среди соматотропином, \%

в пуле пролактинсекретирующих опухолей гипофиза превалируют микроаденомы гипофиза, которые составляют 50-60\% от всех пролактином; в пуле соматотропином преобладают макроаденомы гипофиза, на их долю приходится 79-88\%. В нашу работу были включены только пациенты с макроаденомами, этим можно объяснить различия объемов исследуемых опухолей по сравнению с другим исследованием.
Ранее при анализе данных МРТ пациентов с гормонально неактивными аденомами гипофиза в 49 из 54 случаев $(90,7 \%)$ было отмечено супраселлярное распространение опухоли [8]. В настоящем исследовании, проведенном на бо́льшей когорте пациентов Московской области, супраселлярное направление роста среди гормонально неактивных аденом гипофиза встречалось со схожей частотой $(83,5 \%)$. В исследовании, обобщившем результаты МРТ 297 пациентов с соматотропиномами, отмечалось превалирование инфраселлярного направления роста опухолей гипофиза, частота которого достигала $45,8 \%$ [9]. Согласно нашим данным, инфраселлярное направление роста среди соматотропином отмечалось еще чаще в 66,1\% случаев, и было показано, что это не характерно для опухолей гипофиза других видов.

\section{Заключение}

В когорте пациентов Московской области макроаденомы гипофиза с различной гормональной активностью различались по размерам и характеру распространения за пределы турецкого седла. Эндоселлярное расположение макроаденом гипофиза всех видов встречалось достаточно редко не более чем в 7,8\% случаев. Среди гормонально неактивных макроаденом гипофиза и макропролактином преобладающим было супраселлярное направление роста опухоли $(83,5$ и $79,3 \%$ соответственно); среди соматотропином - инфраселлярное направление $(66,1 \%)$.

Наибольшие средние объемы опухоли отмечались у гормонально неактивных макроаденом гипофиза (6620 мм³), несколько меньшие у пролактином (5365 мм $\left.^{3}\right)$ и соматотропином $\left(3052 \mathrm{mм}^{3}\right)$. Однако индивидуальные показатели объема опухолей гипофиза более $70000 \mathrm{mм}^{3}$ наблюдались именно среди гормонально активных опухолей гипофиза, что необходимо учитывать при планировании дальнейшего обследования. (๕)

\section{Литература}

1. Karavitaki N. Prevalence and incidence of pituitary adenomas. Ann Endocrinol (Paris). 2012;73(2):79-80. doi: 10.1016/j. ando.2012.03.039.

2. Ezzat S, Asa SL, Couldwell WT, Barr CE, Dodge WE, Vance ML, McCutcheon IE. The prevalence of pituitary adenomas: a systematic review. Cancer. 2004;101(3):613-9. doi: 10.1002/cncr.20412.

3. Fernandez A, Karavitaki N, Wass JA. Prevalence of pituitary adenomas: a community-based, cross-sectional study in Banbury (Oxfordshire,
UK). Clin Endocrinol (Oxf). 2010;72(3):377-82. doi: 10.1111/j.1365-2265.2009.03667.x.

4. Tjörnstrand A, Gunnarsson K, Evert M, Holmberg $E$, Ragnarsson $O$, Rosén T, Filipsson Nyström $\mathrm{H}$. The incidence rate of pituitary adenomas in western Sweden for the period 2001-2011. Eur J Endocrinol. 2014;171(4):51926. doi: 10.1530/EJE-14-0144.

5. Fernández-Balsells MM, Murad MH, Barwise A, Gallegos-Orozco JF, Paul A, Lane MA, Lampropulos JF, Natividad I, Perestelo-Pérez L, Ponce de León-Lovatón PG, Erwin PJ, Carey J, Mon- tori VM. Natural history of nonfunctioning pituitary adenomas and incidentalomas: a systematic review and metaanalysis. J Clin Endocrinol Metab. 2011;96(4):905-12. doi: 10.1210/ jc.2010-1054.

6. Orija IB, Weil RJ, Hamrahian AH. Pituitary incidentaloma. Best Pract Res Clin Endocrinol Metab. 2012;26(1):47-68. doi: 10.1016/j. beem.2011.07.003.

7. Famini P, Maya MM, Melmed S. Pituitary magnetic resonance imaging for sellar and parasellar masses: ten-year experience in 2598 patients. J Clin Endocrinol Metab. 
2011;96(6):1633-41. doi: 10.1210/jc.20110168.

8. Дедов ИИ, ред. Клиническая нейроэндокринология. М.: УП Принт; 2001. 343 с.

\section{References}

1. Karavitaki N. Prevalence and incidence of pituitary adenomas. Ann Endocrinol (Paris). 2012;73(2):79-80. doi: 10.1016/j. ando.2012.03.039.

2. Ezzat S, Asa SL, Couldwell WT, Barr CE, Dodge WE, Vance ML, McCutcheon IE. The prevalence of pituitary adenomas: a systematic review. Cancer. 2004;101(3):613-9. doi: 10.1002/cncr.20412.

3. Fernandez A, Karavitaki N, Wass JA. Prevalence of pituitary adenomas: a community-based, cross-sectional study in Banbury (Oxfordshire, UK). Clin Endocrinol (Oxf). 2010;72(3):377-82. doi: 10.1111/j.1365-2265.2009.03667.x.

4. Tjörnstrand A, Gunnarsson K, Evert $M$, Holmberg $E$, Ragnarsson O, Rosén T, Filipsson Nyström $\mathrm{H}$. The incidence rate of pituitary adenomas in western Sweden for the period
9. Potorac I, Petrossians P, Daly AF, Schillo F, Ben Slama C, Nagi S, Sahnoun M, Brue T, Girard N, Chanson $P$, Nasser $G$, Caron $P$, Bonneville $F$, Raverot G, Lapras V, Cotton F, Delemer B, Higel B, Boulin A, Gaillard S, Luca F, Goichot B, Di-

2001-2011. Eur J Endocrinol. 2014;171(4):51926. doi: 10.1530/EJE-14-0144.

5. Fernández-Balsells $M M$, Murad $M H$, Barwise $A$, Gallegos-Orozco JF, Paul A, Lane MA, Lampropulos JF, Natividad I, Perestelo-Pérez L, Ponce de León-Lovatón PG, Erwin PJ, Carey J, Montori VM. Natural history of nonfunctioning pituitary adenomas and incidentalomas: a systematic review and metaanalysis. J Clin Endocrinol Metab. 2011;96(4):905-12. doi: 10.1210/jc.2010-1054.

6. Orija IB, Weil RJ, Hamrahian AH. Pituitary incidentaloma. Best Pract Res Clin Endocrinol Metab. 2012;26(1):47-68. doi: 10.1016/j. beem.2011.07.003. etemann JL, Beckers A, Bonneville JF. Pituitary MRI characteristics in 297 acromegaly patients based on T2-weighted sequences. Endocr Relat Cancer. 2015;22(2):169-77. doi: 10.1530/ ERC-14-0305.

7. Famini P, Maya MM, Melmed S. Pituitary magnetic resonance imaging for sellar and parasellar masses: ten-year experience in 2598 patients. J Clin Endocrinol Metab. 2011;96(6):1633-41. doi: 10.1210/jc.2011-0168.

8. Dedov II, editor. Klinicheskaya neyroendokrinologiya [Clinical neuroendocrinology]. Moscow: UP Print; 2011. 343 p. (in Russian).

9. Potorac I, Petrossians P, Daly AF, Schillo F, Ben Slama C, Nagi S, Sahnoun M, Brue T, Girard N, Chanson $P$, Nasser $G$, Caron $P$, Bonneville $F$, Raverot G, Lapras V, Cotton F, Delemer B, Higel B, Boulin A, Gaillard S, Luca F, Goichot B, Dietemann JL, Beckers A, Bonneville JF. Pituitary MRI characteristics in 297 acromegaly patients based on T2-weighted sequences. Endocr Relat Cancer. 2015;22(2):169-77. doi: 10.1530/ERC-14-0305.

\title{
Growth particulars of pituitary macroadenomas with various hormonal activities
}

\author{
Ilovayskaya I.A. • Dreval' A.V. • Krivosheeva Yu.G. • \\ Astaf'eva L.I. • Stashuk G.A.'
}

Background: Pituitary adenoma is not as infrequent as thought previously. The prevalence of macroadenomas in general population is up to $0.16-0.2 \%$. Magnetic resonance imaging (MRI) is a method of choice in diagnosis of pituitary adenomas. Until now, specifics of imaging of pituitary adenomas with various hormonal activities have not been discussed. Aim: To analyze comparatively the size, volumes and growth direction in pituitary macroadenomas with various hormonal activities. Materials and methods: We analyzed MRI images of 305 patients with hypophyseal adenomas of more than $10 \mathrm{~mm}$ diameter, among them with non-functioning adenomas $(n=109)$, prolactinomas $(n=58)$, and somatotropinomas $(n=138)$. Results: Depending on their hormonal activity, hypophyseal adenomas had different volumes ( $p$ <.001): non-functioning hypophyseal adenomas had the volume of 6620 [2637; 14492] $\mathrm{mm}^{3}$, prolactinomas - $5365[1495 ; 10316] \mathrm{mm}^{3}$, somatotropinomas - $3052[1696 ; 5727] \mathrm{mm}^{3}$. In the majority of patients from all groups, extrasellar growth at several directions was observed. Onedirectional growth was seen in $29 \%$ of non-functioning hypophyseal adenomas, $41 \%$ of prolactinomas and $37 \%$ of somatotropinomas ( $p>0.05$ ). Non-functioning hypophyseal adenomas and prolactinomas demonstrated mostly suprasellar growth (in 83.5 and $79.3 \%$ of cases, respectively), whereas somatotropinomas were growing mostly in infrasellar direction (66.1\%). Conclusion: These characteristic features of hypophyseal macroadenomas with various hormonal activities could be used for differential diagnosis, which may help to optimize patient assessment during the diagnostic work-up.

Key words: hypophyseal macroadenoma, non-functioning hypophyseal adenoma, prolactinoma, somatotropinoma, growth direction of hypophyseal adenoma

\section{doi: 10.18786/2072-0505-2016-44-4-451-456}

Ilovayskaya Irena A. - MD, PhD, Senior Research Fellow, Department of Therapeutic Endocrinology $\triangle$ 61/2-9 Shchepkina ul., Moscow, 129110, Russian Federation. Tel.: +7 (495) 6317492.

E-mail: irena.ilov@yandex.ru

Dreval' Aleksandr V. - MD, PhD, Professor, Head of Department of Therapeutic Endocrinology; Chief of Chair of Endocrinology, Postgraduate Training Faculty $^{1}$

Krivosheeva Yuliya G. - MD, Postgraduate Student, Chair of Endocrinology, Postgraduate Training Faculty $^{1}$

Astaf'eva Lyudmila I. - MD, PhD, Leading Research Fellow, Pituitary Tumor Department ${ }^{2}$

Stashuk Galina A. - MD, PhD, Professor, Chief Research Fellow, Department of Roentgenology; Chair of Radiology, Postgraduate Training Faculty'

'Moscow Regional Research and Clinical Institute (MONIKI); 61/2 Shchepkina ul., Moscow, 129110, Russian Federation

${ }^{2}$ Burdenko Neurosurgical Institute; 16 4-ya Tverskaya-Yamskaya ul., Moscow, 125047, Russian Federation 